Case Report

\title{
Cavitary Lung Cancer lined with Normal Bronchial Epithelium and Cancer Cells
}

\author{
Taichiro Goto ${ }^{1 凶}$, Arafumi Maeshima², Yoshitaka Oyamada ${ }^{3}$, and Ryoichi Kato ${ }^{1}$ \\ 1. Department of General Thoracic Surgery, National Hospital Organization Tokyo Medical Center, Tokyo, Japan \\ 2. Department of Pathology, National Hospital Organization Tokyo Medical Center, Tokyo, Japan \\ 3. Department of Respiratory Medicine, National Hospital Organization Tokyo Medical Center, Tokyo, Japan
}

$\triangle$ Corresponding author: Taichiro Goto, M.D., Department of General Thoracic Surgery, National Hospital Organization Tokyo Medical Center, Meguro-ku, Tokyo 152-8902, Japan. Phone: 81-3-3411-0111; Fax: +81-3-3412-9811; Email: taichiro@1997.jukuin.keio.ac.jp

(c) Ivyspring International Publisher. This is an open-access article distributed under the terms of the Creative Commons License (http://creativecommons.org/ licenses/by-nc-nd/3.0/). Reproduction is permitted for personal, noncommercial use, provided that the article is in whole, unmodified, and properly cited.

Received: 2011.06.13; Accepted: 2011.09.29; Published: 2011.10.04

\begin{abstract}
Reports of cavitary lung cancer are not uncommon, and the cavity generally contains either dilated bronchi or cancer cells. Recently, we encountered a surgical case of cavitary lung cancer whose cavity tended to enlarge during long-term follow-up, and was found to be lined with normal bronchial epithelium and adenocarcinoma cells.
\end{abstract}

Key words: cavitary lung cancer, surgery, histology, bronchial epithelium, traction bronchiectasis.

\section{Case Report}

The patient was a 60 -year-old man with a history of smoking 10 cigarettes per day for 35 years. He was found to have a small shadow in the right lower lung field on chest X-ray in 2006, and was thereafter followed-up at another hospital (Figure 1A). Chest X-ray in 2007 revealed a cavitating shadow at the same site (Figure 1B). A chest X-ray in 2008 showed thickening of the cavity wall, and that in 2009 revealed the tendency of the entire cavity shadow to enlarge (Figure $1 C, D)$. He was referred to our department. Computed tomography showed an inhomogeneous thickening of the cavity wall and spiculation from the tumor margin, as well as the presence of lung structures in the cavity (Figure 2A). Bronchoscopic biopsy of the cavity wall led to a diagnosis of adenocarcinoma. Under a diagnosis of lung cancer (cT2aNOM0), right lower lobectomy with hilar and mediastinal lymph node dissection was performed.
The tumor measured $48 \times 42 \times 36 \mathrm{~mm}$. Gross examination of the tumor showed a cavity whose wall was grayish-white, uneven in thickness, and was torn in some areas (Figure 2B). The tumor had irregular borders, showing spiculation. Interestingly, lung tissue and blood vessels were present in the cavity and were in contact with the extralesional lung through the tears in the cavity wall. Histopathologically, the tumor was composed of atypical bronchial epithelial cells proliferating in a tubular pattern (pT2aN0M0). The internal surface of the cavity wall was lined with dilated bronchi and adenocarcinoma cells, and the extensive area of collapsed scars was observed around the dilated bronchi (Figure 3A-F).

At present, 18 months after surgery, the patient remains free of disease. 


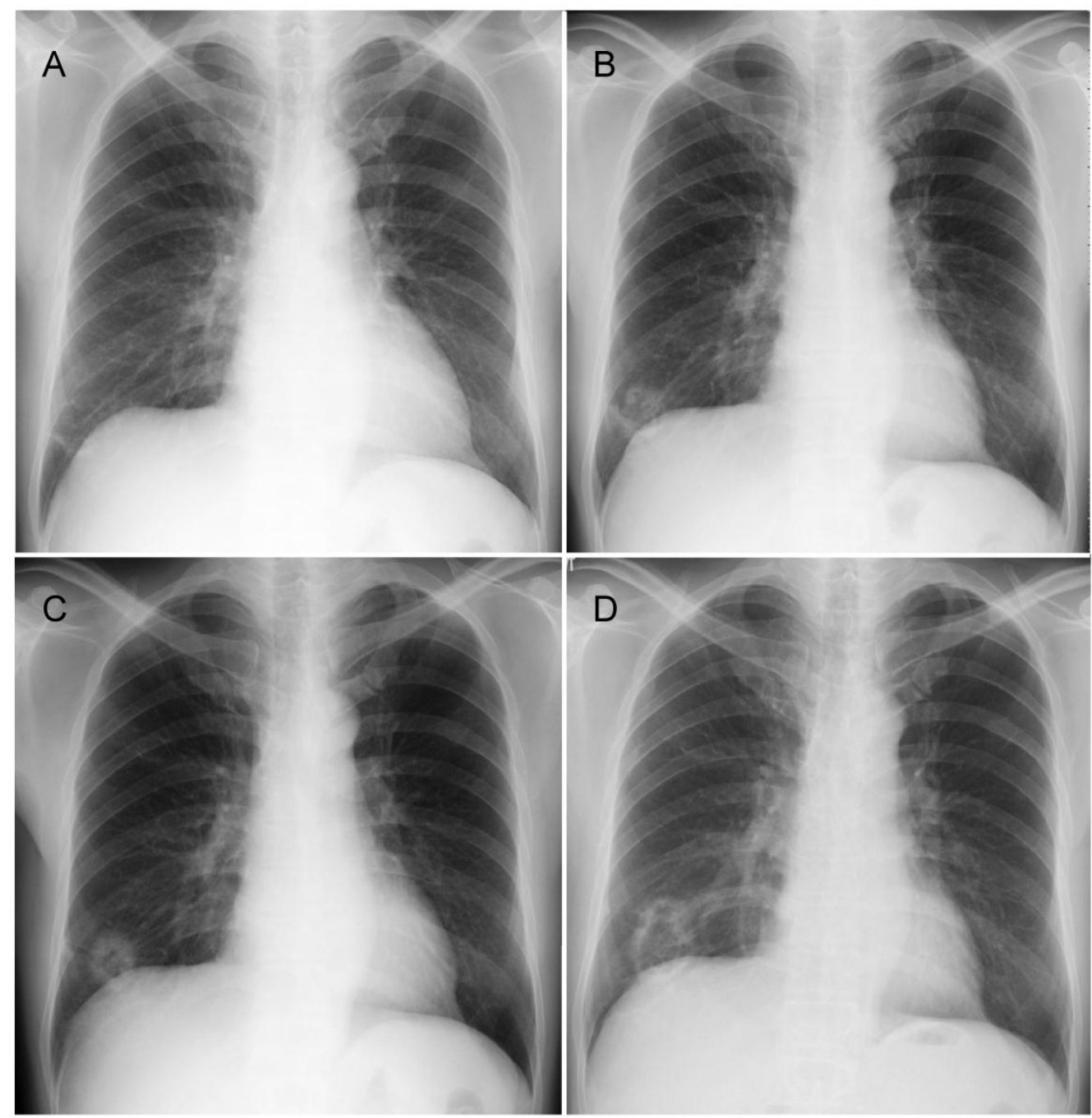

Figure I. Chest X-ray findings. A, B, C, and D show chest X-rays taken in 2006, 2007, 2008, and 2009, respectively. These $X$-rays revealed a lesion in the right lower lung field, which formed a cavity and enlarged over time.
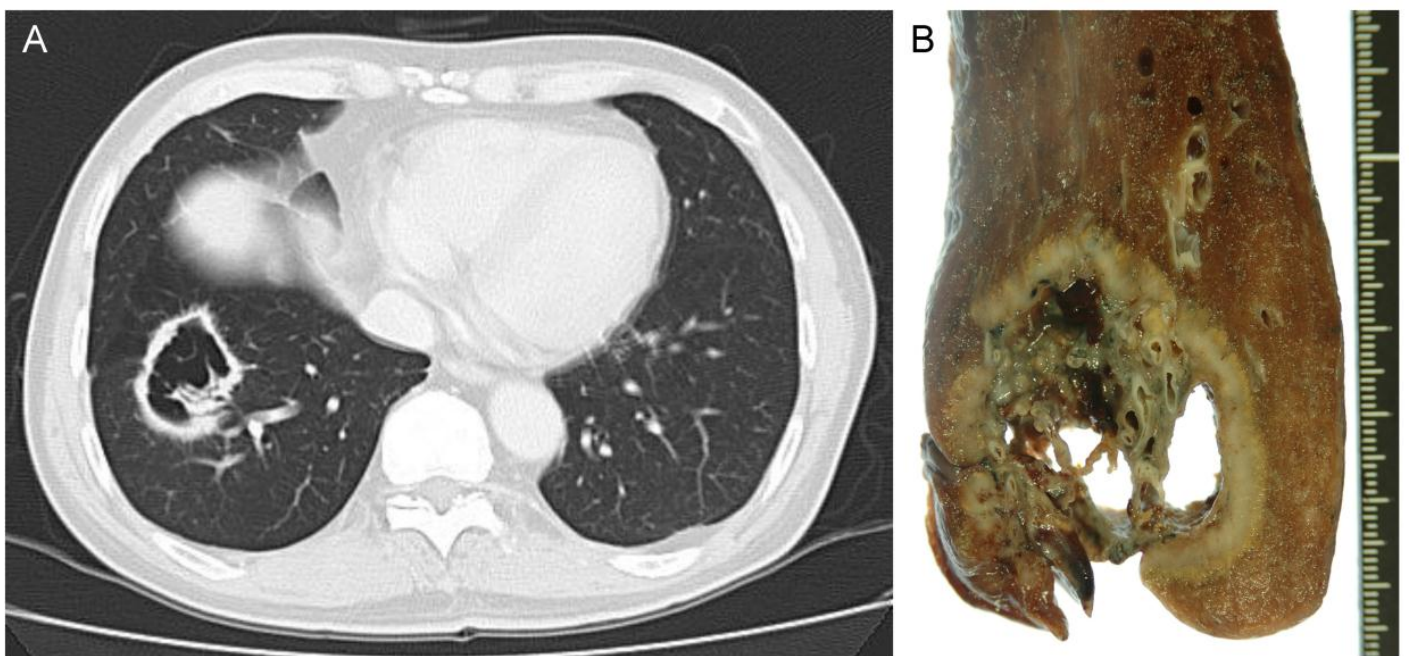

Figure 2. Radiologic and macroscopic findings. A, Computed tomography showed a cavitary shadow in the basal segment of the right lung, and the cavity contained lung tissue. B, Macroscopically, the cavity was torn in some areas, and lung tissue and blood vessels could be observed in the cavity. 

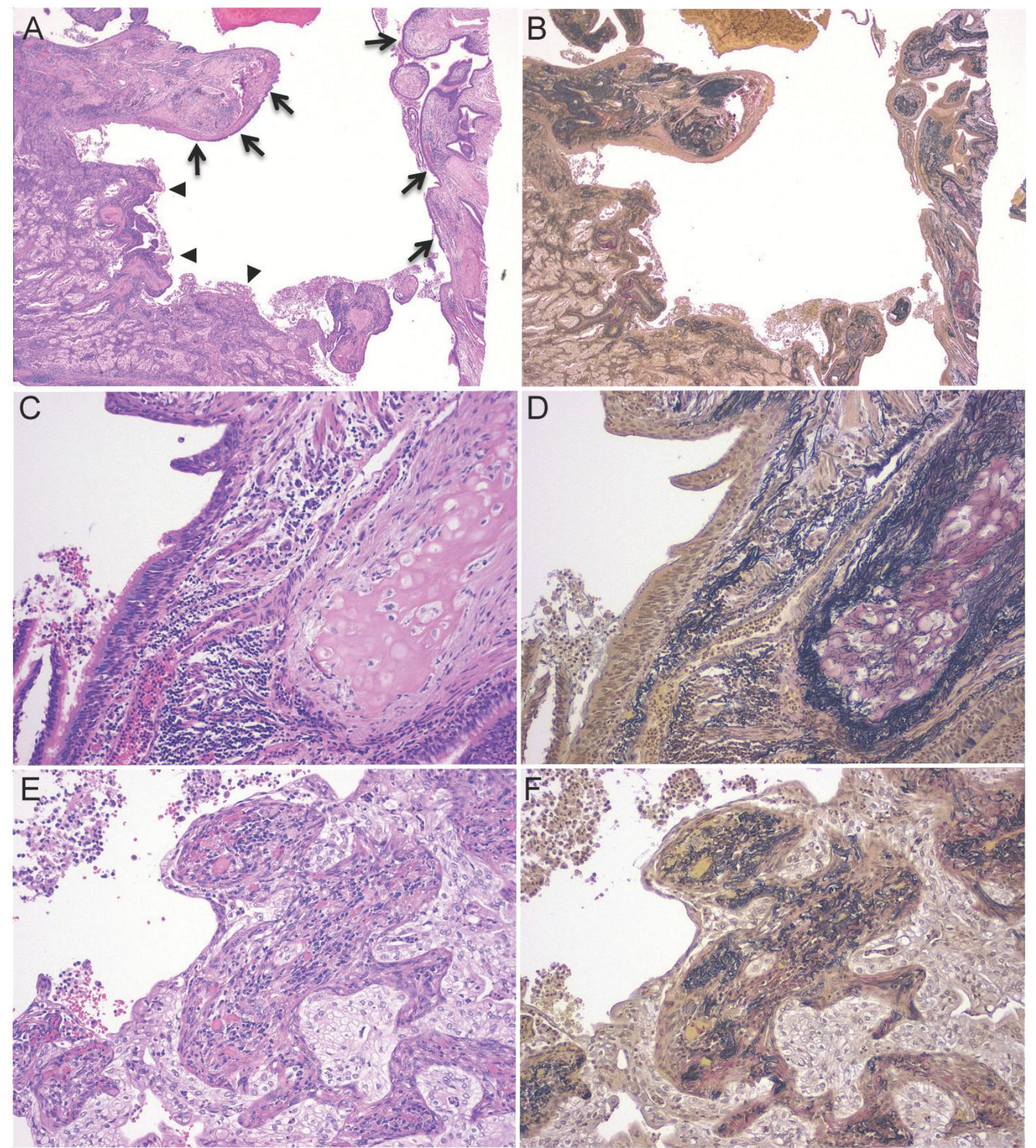

Figure 3. Pathological findings. A-B, The cavity was lined with dilated bronchi (arrow) and tumor cells (arrowhead). The extensive area of collapsed scars was observed around the dilated bronchi. C-D, The portion of the cavity wall lined with normal ciliated bronchial epithelium. Normal bronchial cartilage was observed in the vicinity. E-F, The portion of the cavity lined by tumor tissue. There was collapsed lung in the tumor (A, C, E, Hematoxylin and eosin staining; B, D, F, Elastica-van Gieson staining).

\section{Discussion}

The frequency of cavity formation in primary lung cancer has been reported to be $2-16 \%$, with squamous cell carcinoma and adenocarcinoma ac- counting for $45-63$ and $30-53 \%$, respectively [1]. The possible mechanisms of cavity formation include: i) ischemic necrosis due to occlusion of feeding vessels, ii) check-valve mechanism of the conducting bron- 
chus, iii) elastic traction by the surrounding lung tissue, iv) tumor development in pre-existing lesions such as bullae, and v) neoplastic cell autophagism [2-5].

We speculate that the mechanism of cavity formation in this case was as follows: a scar of collapsed elastic fibers was formed in cancer tissue, resulting in the elastic retraction of the bronchi embedded in the scar, and, during the development of bronchiectasis, the bronchial wall was disrupted in some places, with the result that the tumor tissue shared the cavity wall with the bronchus. A small portion of lung tissue and blood vessels stayed inside the cavity through the tears in the cavity wall during the further development of bronchiectasis.

Cavitary lung cancer which contains lung tissue inside the cavity is a rare entity, but if a tumor shows malignant features on imaging studies, such as wall irregularity, notching, inhomogeneous thickening of the cavity wall, and an enlarging tendency, it is necessary to perform bronchoscopy or surgical biopsy.

\section{Conflict of Interest}

The authors have declared that no conflict of interest exists.

\section{References}

[1] Sugimoto Y, Semba H, Fujii S, et al. [Clinical analysis of primary lung cancer with a thin-walled cavity to explain the mechanism of thin-walled cavity formation]. Nihon Kokyuki Gakkai Zasshi. 2007; 45: 460-464.

[2] Koizumi N, Akita S, Sakai K, et al. Classification of air density areas in CT-pathologic correlation of pulmonary adenocarcinoma. Radiat Med. 1995; 13: 279-284.

[3] Weisbrod GL, Chamberlain D, Herman SJ. Cystic change (pseudocavitation) associated with bronchioloalveolar carcinoma: a report of four patients. J Thorac Imaging. 1995; 10: 106-111.

[4] Weisbrod GL, Towers MJ, Chamberlain DW, et al. Thin-walled cystic lesions in bronchioalveolar carcinoma. Radiology. 1992; 185: 401-405.

[5] Yoshida T, Harada T, Fuke S, et al. Lung adenocarcinoma presenting with enlarged and multiloculated cystic lesions over 2 years. Respir Care. 2004; 49: 1522-1524. 